\title{
Radiopacity and Microhardness Changes and Effect of X-ray Operating Voltage in Resin-Based Materials before and after the Expiration date
}

\author{
Camila Tirapelli $^{\mathrm{a} *}$, Fernanda de Carvalho Panzeri ${ }^{\mathrm{a}}$,Heitor Panzeri, \\ Luiz Carlos Pardini ${ }^{\mathrm{b}}$, Osvaldo Zaniquelli ${ }^{\mathrm{a}}$ \\ ${ }^{a}$ Department of Dental Materials and Prosthodontics - University of São Paulo, \\ School of Dentistry of Ribeirão Preto - SP, Brazil \\ ${ }^{\mathrm{b}}$ Department of Morphology, Stomatology and Physiology - University of São Paulo, \\ School of Dentistry of Ribeirão Preto - SP, Brazil
}

Received:August 21, 2003; Revised: January 25, 2004

\begin{abstract}
This study observed alteration in the radiopacity and microhardness of expired resin-based materials compared to non-expired materials and the operating characteristics of the X-ray source used. Five $2 \mathrm{~mm}$-thick cured specimens were prepared for each material: composite resins (P60®, Z100®), and a compomer (Dyract AP®). Radiopacity of the specimens was evaluated comparing the density of the resin-based material to an equivalent $(\mathrm{mm})$ density of a $99.5 \%$ pure aluminum step wedge using a transmission densitometer. Surface microhardness measurements were carried out using a calibrated Vickers indenter on three different points of the same surface. ANOVA and Tukey tests (pre-set alpha $=0.05$ ) revealed that expired materials showed no significant change in radiopacity. One material (Filtek P60) demonstrated lower radiopacity with lower KVp. Change in microhardness wa s statistically significant for Z100: for this material, the microhardness after expiration was significantly lower than before the expiration date.
\end{abstract}

Keywords: radiopacity, expiration date, composites, operating voltage, microhardness

\section{Introduction}

Resin-based composites are frequently used in dentistry, as both restorations and cements, due to their satisfactory esthetic features and ease of utilization. The radiopacity of these materials is important, since it allows the dentist to differentiate the restorative material from carious lesions, and to evaluate lack of adaptation or excess material along the cervical margins ${ }^{1,2}$. Resin-based materials are radiopaque due to components incorporated into the filler. This filler consists of glass and ceramic particles containing heavy metals, such as aluminum, ytterbium, barium, strontium, and zirconium, which confer radiopacity to the composite ${ }^{3}$. These particles may vary greatly in their concentration and composition, and thus the radiopacity of composites varies as well ${ }^{4}$. In general, composites intended for use in anterior teeth have filler particles that are smaller in size and in lower in concentration than those intended for use in posterior teeth ${ }^{1}$.

According to the International Standardization Organi- zation (ISO) $)^{5}$, a resinous dental material should be at least as radiopaque as the same thickness of pure aluminum. In its Specification \#27, the American Dental Association (ADA) Council on Dental Materials, Instruments \& Equipment ${ }^{6}$ also recommends that these materials should present radiopacity.

Investigations on composites ${ }^{1,7,8}$ focused on aspects related to radiopacity. In according to $\mathrm{Cook}^{9}$ some image-forming factors, such as Kilovoltage ( $\mathrm{KVp})$ may influence the radiopacity of the image. In Brazil, dental X-ray equipment usually operates at 60 or $70 \mathrm{KVp}$.

Soderholm ${ }^{10,11}$ and Soderholm et al. ${ }^{12}$, supported by the stress-corrosion theory, related the leakage of ions from dental composites by storage in water. This phenomenon is probably due to leakage of ions such as silicon, barium, strontium, and sodium into the aqueous medium. Release of such ions could break the chemical bond between the filler particle and the matrix, resulting in reduced radiopac-

*e-mail: catirapelli@forp.usp.br 
ity and diminished mechanical resistance of the material. Microhardness is also important for mechanical success of the restoration.

This investigation was based on the chemical adhesion between filler particles and matrix, before and after the expiration date. Alterations on the radiopacity of the material would be representative of lack of union between filler particles and matrix. Incomplete polymerization of the resinbased materials could facilitate debonding between filler and matrix. Microhardness was evaluated to measure the degree of polymerization achieved ${ }^{13}$.

The shelf life of a material is the period, from the date of manufacturing, for which a material retains the physical and mechanical properties necessary to accomplish its prescribed purpose $^{14}$. Properties that change over three to four years may not be necessarily clinically noticeable, but may impact the longevity of the restoration ${ }^{15,16}$. Also, it should be noted that if radiopacity decreases past the expiration date, the diagnostic features of the product would be negatively affected. Although the utilization of expired dental materials is not recommended, the properties of the product affected by aging are still unclear This knowledge is important, since it may allow extension in the recommended shelf life of these expensive materials.

\section{Purpose}

The hypothesis of this study was based on the possibility that the high atomic number elements in the filler particles diffused into the uncured monomer materials over time. When cured and placed into water, an increased rate of loss of these elements was expected, because of the greater surface area of the diffusible mass. The debonding of filler particles caused by the leakage of such ions, would have an impact on optical and mechanical properties, such as radiopacity and microhardness ${ }^{17,18}$.

This study compared the radiopacity and microhardness of composite materials before, as well as at least 6 months after the printed expiration date. Also, variation in radiopacity of these materials was examined when using X-ray machines with different operating voltages.

\section{Material and Methods}

Specimens of composite and compomer (Table 1) were made using circular metallic molds ( $2 \mathrm{~mm}$ thick and $7 \mathrm{~mm}$ in diameter).

Materials were handled according to manufacturer's instructions. After placing the matrix on a glass plate, the material was inserted and pressed flat with a glass plate, in order to make the uncured mass conform to the confines of the mold. The material was then cured using a halogen lamp $\alpha$ for $20 \mathrm{~s}$ each, on the top and bottom surfaces (through the glass plate). Sixty specimens were made, five from each com- mercial brand of material, before and at least 6 months past the expiration date for each test, radiopacity or microhardness. After being manually removed from the matrix, specimens were polished $\beta$ to simulate a clinical situation and to expose underlying composite material to the leaching capacity of the storage medium. The specimens were stored for 7 days in sealed containers with distilled water at room temperature and away from direct light.

For radiopacity evaluation, samples were radiographed using two different types of X-ray instruments: $60 \mathrm{KVp}$, $10 \mathrm{~mA} \chi(200 \mathrm{~ms})$ and $70 \mathrm{KVp}, 8 \mathrm{~mA} \delta(300 \mathrm{~ms})$. A $99.5 \%$ pure aluminum step wedge, with eight different degrees of thickness ( $2 \mathrm{~mm}$ to $16 \mathrm{~mm}$ ) was placed on top of a periapical radiographic film $\theta$. Specimens were placed clockwise, following a randomly pre-established sequence - Dyract, Filtek P60, and Z100 specimens - below the aluminum step wedge. Letters and numbers codified equipment voltage, length of exposure, the kind of material, and the repetition number.

Films were manually developed in a dark room following the time/temperature recommendations of the manufacturer. The optical density of the radiographic images was measured $\phi$, for each of the restorative materials and each different thickness, using the aluminum wedge as reference. Therefore, the optical density value obtained for each material was converted into the relative percentage to $2 \mathrm{~mm}$ of aluminum: $\mathrm{R}=\mathrm{OD} \times 100 \mathrm{OD}(\mathrm{Al})$; where: $\mathrm{R}=$ radiopacity of the restorative material specimen $(2 \mathrm{~mm}), \mathrm{OD}=$ optical density of the specimen $(2 \mathrm{~mm})$, and $\mathrm{OD}(\mathrm{Al})=$ optical density of aluminum $(2 \mathrm{~mm})$.

Surface microhardness measurements were carried out by means of a Vickers Microhardness tester $\omega$ in three different points on the same surface of each sample.

Values obtained were analyzed by ANOVA - Analysis of Variance $(\mathrm{p}<0,05)$ and Tukey test.

\section{Results}

Statistical analysis showed no difference in radiopacity (Table 2) between expired materials and those used before the expiration date. The only material showing a significant difference in radiopacity when using different X-ray units was Filtek $\mathrm{P} 60 \AA$ For this material, values were significantly higher when the material was radiographed using a $70 \mathrm{KVp}$ instrument $(0.64 \pm 0.02)$, compared to a $60 \mathrm{KVp} \mathrm{X}$-ray unit $(0.54 \pm 0.04)$.

Changes in microhardness (Table 3 ) were statistically significant for Z100. For this material, microhardness val-

$\alpha$ Ultralux Electronic®, Dabi Atlante, Ribeirão Preto, SP, Brazil. $\beta$ Sof-Lex ${ }^{\circledR}, 3 \mathrm{M}$ of Brasil, Sumaré, SP, Brazil.

$\chi$ Heliodent 60B® - Siemens, São Paulo, Brazil.

$\delta$ Spectro 70X® - Dabi Atlante, Ribeirão Preto, SP, Brazil.

$\theta$ Kodak Insight Periapical 150, USA.

$\phi$ Vitoreen Photodensitometer - Model 7-424 - USA.

$\omega$ Microhardness Tester - Shimadzu, Japan. 
Table 1. Products, brand, source, lot, and expiration date of the materials studied.

\begin{tabular}{|c|c|c|c|c|c|c|}
\hline Product & Brand & Shade & Source & Lot & Expiration & After expiration** \\
\hline Composite & $\mathrm{Z} 100 ®$ & $\mathrm{C} 2$ & $\begin{array}{l}\text { 3M Dental Products, } \\
\text { St. Paul, MN, USA }\end{array}$ & $\begin{array}{l}9 \mathrm{ET} \\
2 \mathrm{TF}\end{array}$ & $\begin{array}{l}\text { Jul/2002* } \\
\text { Jun/2005 }\end{array}$ & 8 \\
\hline Composite & Filtek P60® & $\mathrm{C} 2$ & $\begin{array}{l}\text { 3M Dental Products, } \\
\text { St. Paul, MN, USA }\end{array}$ & $\begin{array}{l}9 \mathrm{CB} \\
2 \mathrm{NJ}\end{array}$ & $\begin{array}{l}\mathrm{Aug} / 2002 * \\
\mathrm{Jul} / 2005\end{array}$ & 7 \\
\hline Compomer & Dyract ${ }^{\circledR A P}$ & B1 & $\begin{array}{l}\text { Dentsply - DeTrey, } \\
\text { Konstanz, Germany }\end{array}$ & $\begin{array}{l}9904000598 \\
0111001056\end{array}$ & $\begin{array}{c}\mathrm{Feb} / 2001 * \\
\mathrm{Oct} / 2003\end{array}$ & 25 \\
\hline
\end{tabular}

*Materials used after the expiration date.

**Months past the expiration date.

Table 2. Radiopacity of specimens (compared to the same thickness of aluminum) from expired and non-expired materials, radiographed with X-ray units operating in 70 and $60 \mathrm{KVp}$.

\begin{tabular}{lcccccc}
\hline & \multicolumn{3}{c}{ EXPIRED materials } & & \multicolumn{3}{c}{ NON-EXPIRED materials } \\
& Dyract & Z100 & P60 & Dyract & Z100 & P60 \\
\hline $60 \mathrm{KVp}$ & 0.50 & 0.55 & 0.49 & 0.48 & 0.54 & 0.54 \\
& $( \pm 0.06)$ & $( \pm 0.04)$ & $( \pm 0.01)$ & $( \pm 0.03)$ & $( \pm 0.03)$ & $( \pm 0.04)$ \\
$70 \mathrm{KVp}$ & 0.57 & 0.58 & $0.56^{*}$ & 0.56 & 0.54 & $0.64^{*}$ \\
& $( \pm 0.04)$ & $( \pm 0.02)$ & $( \pm 0.01)$ & $( \pm 0.04)$ & $( \pm 0.02)$ & $( \pm 0.02)$ \\
\hline
\end{tabular}

Mean \pm 1 standard deviation, $\mathrm{n}=5$ specimens per group.

*Statistically significant difference.

Table 3. Vickers microhardness values for expired and non-expired materials.

\begin{tabular}{lccccc}
\hline & EXPIRED materials & \multicolumn{4}{c}{ NON-EXPIRED materials } \\
\hline Dyract & Z100 & P60 & Dyract & Z100 & P60 \\
39.7 & 39.7 & 98.5 & 48.8 & 106 & 86.8 \\
46.8 & 46.8 & 90.7 & 41.5 & 107 & 85.0 \\
33.3 & 33.3 & 101.0 & 43.8 & 104 & 81.7 \\
44.1 & 44.1 & 91.0 & 57.1 & 102 & 83.7 \\
48.8 & 48.8 & 87.2 & 39.1 & 104 & 101 \\
$42.54( \pm 6.18)$ & $95.74( \pm 3.95)$ & $93.68( \pm 5.807)$ & $46.06( \pm 7.13)$ & $104.6( \pm 7.13) *$ & $87.64( \pm 7.69)$ \\
\hline
\end{tabular}

*Statistically significant.

ues were significantly lower past the expiration date. This suggests a lower degree of polymerization of the expired material $(95.74 \pm 3.95)$ compared to the unexpired material $(104.6 \pm 3.95)$. Dyract showed lower values for microhardness when expired, but this alteration was not statistically significant. For Filtek P60, microhardness values were higher in the expired material, but this difference was also not statistically significant.

\section{Discussion}

Several studies ${ }^{10-12}$ reported reduction on the filler particles of restorative composites kept in liquid medium for certain periods. The mechanical properties of light-cured composites are also highly dependent on the concentration of the filler particles in the matrix and the degree of polymerization. This is influenced by the concentration of the photo- initiator ${ }^{18}$ that would suffer degradation throughout the shelf life of the material.

In this study, there was no statistically significant change in radiopacity related to expiration for any material tested. It is important to note that the samples made of expired material were not produced immediately past the expiration date: specimens were made a minimum of six months after the material had expired. Differences between the values of radiopacity and microhardness for materials past the expiration date, compared to those not expired, could be expected. However, in this investigation, the storage conditions ( 7 days at room temperature) used were different from those used by Soderholm ${ }^{10,11}$ and Soderholm et al. ${ }^{12}$, in which storage time was greater than seven days. This difference may explain the lack of changes on radiopacity in the present study.

According to $\mathrm{Cook}^{9}$, the kilovoltage of X-ray units pro- 
duces significant alterations in the radiopacity of resin-based materials. This finding is in disagreement with most results obtained in the present study. However, Cook $^{9}$ used X-ray units with kilovoltage varying between 40 and $90 \mathrm{KVp}$. The small difference in kilovoltage of the X-ray units used in this study ( 60 and $70 \mathrm{KVp}$ ) may explain the fact that alteration of radiopacity was observed in only one of the groups (Filtek P60). For this material, radiopacity was higher when the material was radiographed using a $70 \mathrm{KVp}$ instrument. This observation can be explained by the greater absorbance of $\mathrm{X}$-rays from the $70 \mathrm{KV}$ p equipment by Filtek P60. According to $\mathrm{Cook}^{9}$, radiopacity (compared to the same thickness of aluminum) is quite sensitive to the X-ray operating voltage, especially for highly radiopaque materials, however, variation of radiopacity among materials was not a goal of the present investigation.

Hondrum ${ }^{16}$ documented changes in mechanical properties of water-based cements over time (84 months). The hardness values increase significantly after 36 months for zinc phosphate and zinc polycarboxylate, and decrease for glass ionomer. The microhardness data of this study is statistically significant only for Z100, which showed lower values when expired. The findings of Hondrum ${ }^{16}$ were obtained under different storage conditions, and the materials tested were not resin-based. The change in microhardness of Z100 may be explained by incomplete cure of the polymer, perhaps due to degradation, over time, of the components involved in the polymerization of this material. The lack of significant changes in Dyract and Filtek P60 may be due to the short time past after the expiration date, and also to the storage conditions (following manufacturers' recommendations) of all tested materials until their use.

Radiopacity and mechanical properties are only two of the several desirable features of a dental material. Other factors related to the aging process should also be investigated.

Based on the conditions imposed in this study, the following conclusions may be made:

- Radiopacity of resin-based material does not change, at least six months after the expiration date provided by the manufacturer, for Filtek P60®, Dyract, and Z100â composites.

- Radiopacity of the resin-based materials evaluated did not vary when specimens were radiographed using X-ray units with 60 or $70 \mathrm{KVp}$, except for a single material (Filtek P60), which demonstrated increased radiopacity with increased $\mathrm{KV}$.

- Microhardness in resin-based materials presented statistically significant changes only for Z100. When expired, this material presented lower microhardness values than before the expiration date.

\section{References}

1. Toyooka, H; Taira, M; Wakasa, K; Yamaki, Fujita, M;
Wada, T. Radiopacity of 12 visible-light-cured dental composite resins, J. Oral Rehabil, v. 20, p. 615-22, 1993.

2. Rubo, MHM; El-Mowafi. Radiopacity of dual-cured and chemical-cured resin-based cements, Int. J. Prosthodont., v. 18, p. $70-74$.

3. Watts, DC. Radiopacity $\times$ Composition of some barium and strontium glass composites, J. Dent., v. 15, p. 38-43, 1987.

4. Hosoda, H; Yamada, T; Inokoshi, S. SEM and elemental analysis of composite resins, J. Prosthet. Dent., v. 64, p. 669, 1990.

5. ISO: DP 4049. Dental resin-based restorative materials. International Standards Organization, clause 6.10, 1985.

6. American Dental Association Council on Dental Materials, Instruments and Equipments. The desirability of using radiopaque plastics in dentistry: a status report, J. Am. Dent. Assoc., v. 102, p. 347, 1981.

7. Van Dijken, J.W.V.; Wing, K.R.; Ruyter, E. An evaluation of the radiopacity of composite restorative materials used in class I and class II cavities, Acta Odontol. Scand., v. 47, p. 401-407, 1989.

8. Hara, A.T.; Serra, M.C.; Haiter-Neto, F.; Rodrigues, S.Jr.; AL. Radiopacity of esthetic restorative materials compared with human tooth structure, Am. Dent. J., v. 14, p. 383-386, 2001.

9. Cook, W.D. An investigation of the radiopacity of composite restorative materials, Austr. Dent. J., v. 26, p. 105112, 1981.

10. Söderholm, K-J.M. Degradation of glass filler in Experimental Composites, J. Dent. Res., v. 60, p.1867-75, 1981.

11. Söderholm, K-J.M. Leaking of fillers in dental composites, J. Dent. Res., v. 62, p. 126-30, 1983.

12. Söderholm, K-J.M.;Zigan, M.; Ragan, M.; Fischlschweiger, W.; Bergman, M. Hydrolytic degradation of dental composites, J. Dent. Res., v. 63, p. 1248, 1984.

13. McKinney J.E.; Antonucci, J.M.; Rupp, N.W. Wear and microhardness of glass ionomer cements, J. Dent. Res., v. 66, p. 1134-39, 1987.

14. National Standards Institute/ American Dental Association Specification $\mathrm{n}^{\circ} 33$, Dental Terminology. Chicago: American Dental Association

15. Hondrum, S.O. The U. S. Army Institute of dental Research dental materials shelf-life survey: questionnaire results, Mil Med, v.156, p. 488-91, 1991.

16. Hondrum, S.O. Storage stability of dental luting agents. J. Prosthet. Dent., v. 81, p. 464-48, 1999.

17. Söderholm, K-J.M. Relationship between compressive yield strength and filler fractions of PMMA composites, Acta Odontol. Scan., v. 40, p. 145-150, 1982.

18. Krishnan, V.K.; Yamuna, V. Effect of initiator concentration, exposure time and particle size of the filler upon the mechanical properties of light-curing radiopaque dental composite, J. Oral Rehabil, v. 25, p. 747-51, 1998. 